Lichnerowicz, A. (1983). Quantum mechanics and deformations of geometrical dynamics, in Quantum Theory, Groups, Fields and Particles, A. O. Barut, ed., Reidel, Dordrecht.

Liu, K. C. (1976). Journal of Mathematical Physics, 17, 859.

Lu, Z. (1992). Journal of Mathematical Physics, 33, 446.

Lukin. M., et al. (1993). Lagrangian and Hamiltonian formalism on a quantum plane, University of Alabama preprint, UAHEP-931.

Majid, S. (1992). The quantum double as quantum mechanics, DAMPT/92-48.

Malik, R. P. (1993). Physics Letters B, 316, 257.

Manin, Yu. I. (1988). Quantum groups and non-commutative geometry, Montreal University preprint CRM-1561.

Moyal, J. E. (1949). Proceedings of the Cambridge Philosophical Society, 45, 99.

Patera, J., and Zassenhaus, H. (1988). Journal of Mathematical Physics, 29, 665.

Remler, A. E. (1975). Annals of Physics, 95, 455.

Rieffel, M. A. (1988). Canadian Journal of Mathematics, 40, 257.

Rieffel, M. A. (1989). Communications in Mathematical Physics, 122, 531.

Rivier, D. C. (1951). Physical Review, 83, 852.

Schwinger, J. (1960). Proceedings of the National Academy.of Sciences of the USA, 46, 570, 893.

Schwinger. J. (1961). Proceedings of the National Academy of Sciences of the USA, 47, 1075.

Schwinger, J. (1970). Quantum Kinematics and Dynamics, Benjamin, New York.

Sharan, P. (1979). Physical Review D, 20, 414.

Sudbery, A. (n.d.). Quantum differential calculus and Lie algebras, PRE-33667-York.

Vey, J. (1974). Commentarii Mathematici Helvetici, 50, 421.

Weyl, H. (1931). Theory of Groups and Quantum Mechanics, Methuen, London.

Wigner, E. P. (1932). Physical Review, 40, 749.

Woronowicz, S. L. (1987). Communications in Mathematical Physics, 111, 613.

\title{
Erratum
}

\section{Quantum Mechanics as a Matrix Symplectic Geometry}

\author{
A. E. F. Djemai
}

The following corrections should be made to the above article that was published in International Journal of Theoretical Physics, 35, 519-556 (1996).

The top of page 548 should read:

It follows that the quantities $S_{\overrightarrow{m n}}^{\vec{p}_{-}}$are given by:

$$
S_{\vec{m} n}=\exp \left[i \alpha_{2}(\vec{m}+\vec{n}, \vec{p})\right] \cos \left[i \alpha_{2}(\vec{m}, \vec{n})\right] Y_{\vec{m}+\vec{n}-\vec{p}}-\delta_{-\vec{m}+\vec{n}, \overrightarrow{0}} Y_{-\vec{p}}
$$

Finally, we have the following multiplication law:

$$
\begin{aligned}
Y_{\vec{m}} \cdot Y_{\vec{n}} & =\exp \left[i \alpha_{2}(\vec{n}, \dot{\vec{m}})\right] Y_{\vec{m}+\vec{n}} \\
& =K_{\vec{m} n} 1+\left(S_{m \vec{n}}^{\vec{p}}+1 / 2 C \vec{m}_{\vec{m} n}^{\vec{n}}\right) Y_{\vec{p}}
\end{aligned}
$$

